\title{
Young's double-slit experiment with single photons and quantum eraser
}

\section{Citation}

Rueckner, Wolfgang, and Joseph Peidle. 2013. “Young's Double-Slit Experiment with Single Photons and Quantum Eraser." American Journal of Physics 81 (12): 951. doi:10.1119/1.4819882.

\section{Published Version}

10.1119/1.4819882

\section{Permanent link}

http://nrs.harvard.edu/urn-3:HUL.InstRepos:27413728

\section{Terms of Use}

This article was downloaded from Harvard University's DASH repository, and is made available under the terms and conditions applicable to Other Posted Material, as set forth at http:// nrs.harvard.edu/urn-3:HUL.InstRepos:dash.current.terms-of-use\#LAA

\section{Share Your Story}

The Harvard community has made this article openly available.

Please share how this access benefits you. Submit a story.

Accessibility 


\title{
APPARATUS AND DEMONSTRATION NOTES
}

The downloaded PDF for any Note in this section contains all the Notes in this section.

Frank L. H. Wolfs, Editor

Department of Physics and Astronomy, University of Rochester, Rochester, New York 14627

This department welcomes brief communications reporting new demonstrations, laboratory equipment, techniques, or materials of interest to teachers of physics. Notes on new applications of older apparatus, measurements supplementing data supplied by manufacturers, information which, while not new, is not generally known, procurement information, and news about apparatus under development may be suitable for publication in this section. Neither the American Journal of Physics nor the Editors assume responsibility for the correctness of the information presented.

Manuscripts should be submitted using the web-based system that can be accessed via the American Journal of Physics home page, http://ajp.dickinson.edu and will be forwarded to the ADN editor for consideration.

\section{Young's double-slit experiment with single photons and quantum eraser}

\author{
Wolfgang Rueckner ${ }^{a)}$ and Joseph Peidle ${ }^{\text {b) }}$ \\ Harvard University, Cambridge, Massachusetts 02138
}

(Received 25 January 2012; accepted 19 August 2013)

\begin{abstract}
An apparatus for a double-slit interference experiment in the single-photon regime is described. The apparatus includes a which-path marker that destroys the interference as well as a quantum eraser that restores it. We present data taken with several light sources, coherent and incoherent and discuss the efficacy of these as sources of single photons. (C) 2013 American Association of Physics Teachers. [http://dx.doi.org/10.1119/1.4819882]
\end{abstract}

\section{INTRODUCTION}

This apparatus note is a report on a completely updated and much improved double-slit demonstration experiment previously published in this journal. ${ }^{1}$ The (extremely) expensive, specialized, and hard-to-find electronic instrumentation has been replaced by a CCD and a computer. The apparatus has been scaled up in size so that the system parameters can be varied. In particular, the demonstration has been expanded to include a which-path marker and a quantum eraser-one can erase the which-path information to recover interference. ${ }^{2}$ The act of measurement and the design of the experiment affect what is being measured. Even if not actually measured, the mere possibility that an observer could determine which slit the photon passed through causes the interference pattern to switch to non-interference. ${ }^{3}$ This experiment nicely demonstrates this aspect of quantum mechanics and complements discussions of non-local effects of detectors and particle correlations hidden in noise.

\section{THE DEMONSTRATION EXPERIMENT}

\section{A. Double-slit interference}

In explaining the single-photon experiment, it is assumed that students are familiar with the classical Young's doubleslit experiment. The geometry of the quantum version of the experiment is the same and thus will need little elaboration. A double-slit is located in the middle of a long, light-tight PVC pipe. An extremely narrow entrance slit at one end of the pipe lets light in, and an Electron Multiplying CCD (EMCCD) camera at the opposite end records the double-slit interference pattern, one photon at a time. Thus, it can be shown that the interference is not between photons, but rather that each photon interferes only with itself due to the quantum uncertainty of which path through the optical apparatus it takes. What we observe as an interference pattern is the accumulated sum of many single photon interference events. The experiment can be performed with any kind of light source, including an incandescent lamp (polarized or not).

\section{B. Which-path experiment introduction}

Students are not likely to be familiar with the which-path experiment and we find it useful to first perform the experiment with a laser in the usual manner: the laser, double-slit, and polarizers are all mounted on an optics rail, visible to the audience, and the interference pattern is projected onto a screen. The images on the screen can all be explained on the basis of the classical wave nature of light. When two, mutually perpendicular polarizing filters are positioned in front of the double-slit, with each filter covering only one slit, the double-slit interference pattern disappears because the waves emanating from the slits are orthogonal to each other and their amplitudes cannot add constructively or destructively. One only sees a single-slit pattern on the screen. Holding a third polarizing filter in front of the screen restores the double-slit pattern if this filter is oriented at $45^{\circ}$ with respect to the other two filters. The third filter selects the same polarization component from the other two filters, making it once more possible to add the amplitudes of the waves. The reduced intensity is consistent with Malus's law.

For this experiment to work properly, the laser's polarization direction should be at $45^{\circ}$ with respect to the polarization direction of the two filters in front of the slits to ensure that the light 


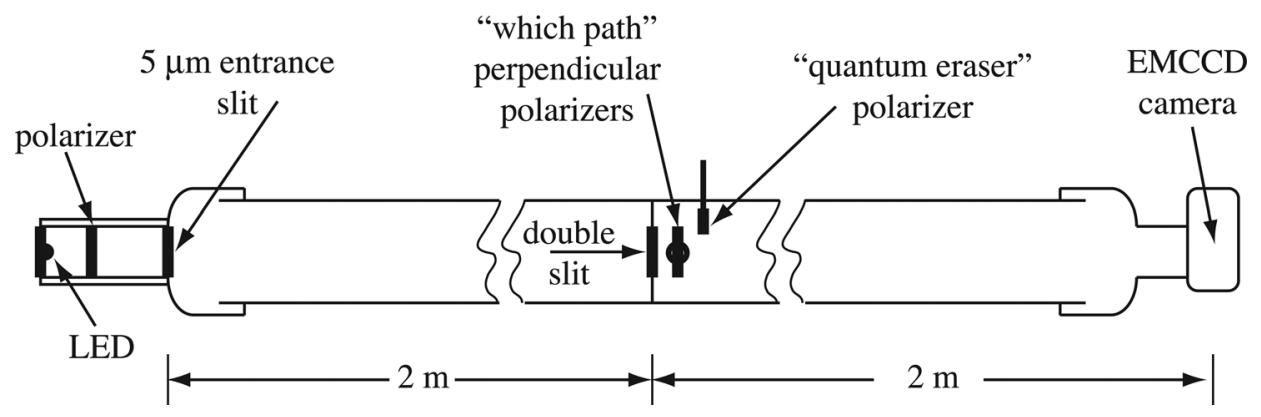

Fig. 1. Layout of the apparatus. A 4 -m long $\times 10-\mathrm{cm}$ diameter PVC pipe supports all optics and acts as a light shield. Light source and camera are mounted on the pipe end-caps.

intensities coming from these slits are the same; otherwise, one cannot secure complete interference. ${ }^{4}$ If an unpolarized laser is used, the experiment can still be conducted by placing a linear polarizing filter oriented at $45^{\circ}$ in front of the laser. Students could be assigned a similar lab or "home" experiment. ${ }^{5}$

\section{The single photon which-path and quantum-eraser experiment}

The classical wave nature of light can no longer describe what happens when the experiment is repeated with a singlephoton light source. For single photons, the double-slit interference pattern can be made to disappear by using a marker. As in the classical wave experiment described above, the marker consists of two, mutually perpendicular, polarizing filters placed in front of the double-slit. Each filter covers only one slit and "marks" the photon passing through that slit with its polarization. A single-slit pattern is all that remains. One does not need to actually measure the photon's polarization state to determine which path it took to reach the detector. The mere fact that the which-path information is available is enough to destroy the interference pattern. By placing a third polarizer, oriented at $45^{\circ}$ with respect to the other two polarizers, before the camera, the double-slit interference pattern is once more restored! All photons emerging from the third polarizer have the same polarization state and thus the which-path information is erased; the third polarizer is the quantum eraser.

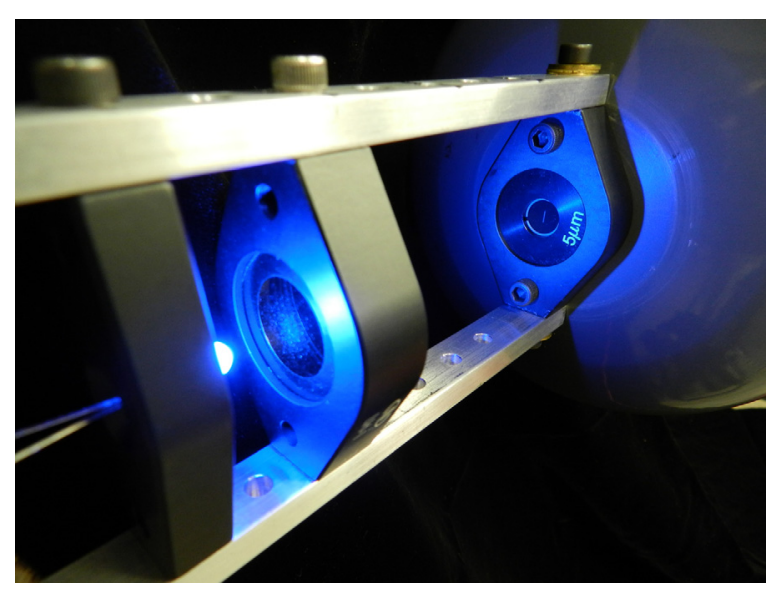

Fig. 2. The blue LED (left), linear polarizer (middle), and 5- $\mu$ m entrance slit (right) are all mounted in standard lens holders and the entire assembly is attached to the end-cap of the PVC pipe.

\section{APPARATUS DETAILS}

Figure 1 illustrates the layout of the apparatus and Figs. 2-4 show some of the details. A 4-m long $\times 10$-cm diameter PVC pipe supports all the optics and acts as a light shield.

\section{A. Photon detector}

The expensive and obsolete vidicon camera used in the previous apparatus has been replaced with a thermoelectrically cooled EMCCD, providing single photon detection sensitivity with a QE of $50 \% .^{6}$ The image area measures $6.58 \times 4.96 \mathrm{~mm}\left(1 / 2^{\prime \prime}\right.$ format $)$ with $658 \times 496$ active pixels. The image data are downloaded through the USB output of the camera to a PC and integrated with software. ${ }^{7}$ The PC displays the interference pattern as it accumulates over a period of time. A graph of the intensity distribution as well as the pixel counts is included in the display. This is a nice feature because it gives a better sense of the signal to background ratio, which is not so obvious in the image. Noise reduction and display options are discussed in Appendix A.

\section{B. Double slit}

The slits are $3 \mathrm{~mm}$ long, precision double air slits, custom ordered. ${ }^{8}$ There are two parameters to work with: the slit separation and the slit width. Because the quantum eraser part of the experiment involves butting two polarizing filters together and

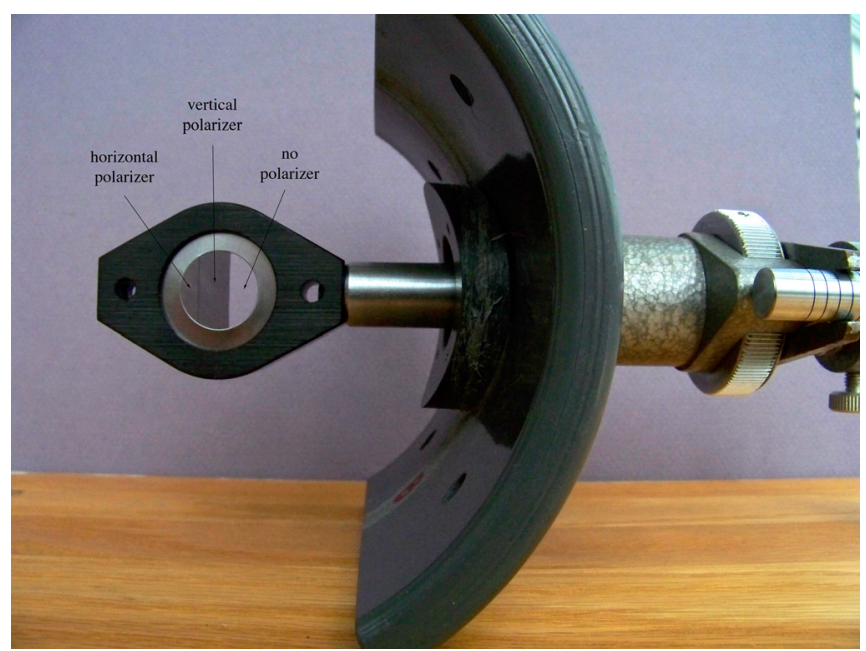

Fig. 3. The which-path marker (left) consists of vertical and horizontal polarizers held by a standard lens mount; its position is adjusted from outside the PVC pipe. 


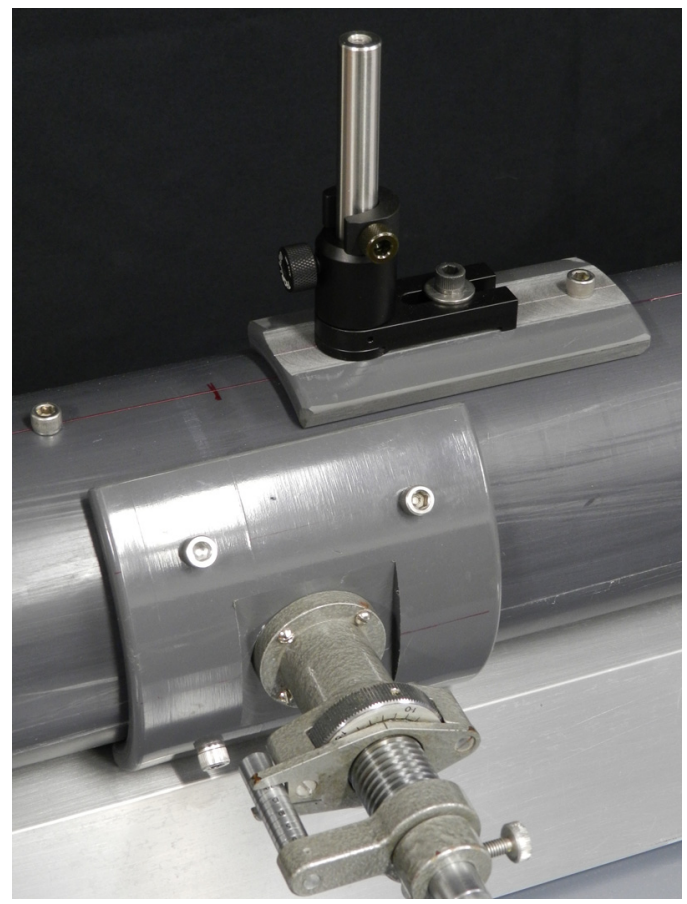

Fig. 4. Both the marker and eraser assemblies attached to the PVC pipe. The eraser polarizer is moved into place by simply lowering the lens post. No fine adjustments are necessary.

has each filter cover only one slit, a slit separation of $1.0 \mathrm{~mm}$ was chosen. Although a slit separation greater than $1.0 \mathrm{~mm}$ would make it easier to cover each slit with a separate filter and would provide a greater number of interference fringes in the image area of the CCD, the distance between the doubleslit and the CCD would become inconveniently large if one wishes to be in the far field region. The "boundary" between the near and the far field regions is given by ${ }^{9}$

$$
L_{\text {screen }} \approx \frac{d^{2}}{\lambda},
$$

where $d$ is the slit separation. For $\lambda=500 \mathrm{~nm}$ and $d=1 \mathrm{~mm}$, the distance $L_{\text {screen }}$ is approximately $2 \mathrm{~m}$. We found that increasing the slit distance from $50 \mathrm{~cm}$ to $2 \mathrm{~m}$ indeed made the interference pattern much cleaner and sharper, in the sense that the light intensity goes to zero in the destructive interference regions. At a distance of $50 \mathrm{~cm}$, the interference pattern appears as a messy convolution of Fresnel and Frauenhoffer diffraction. One could work at closer distances by employing a converging lens and maintain a large "effective" $L_{\text {screen, }}$ but we did not want to complicate the experiment with additional optics.

The finite slit width is responsible for modulating the double-slit interference pattern with a single-slit pattern. When the double-slit pattern is made to go away in the which-path experiment, it is nice to still have a recognizable single-slit diffraction pattern left in its place to be able to differentiate what one observes from background. A slit width of $200 \mu \mathrm{m}$ was chosen so that the image area of the CCD spans the entire central maximum of the single-slit pattern at a distance of $2 \mathrm{~m}$.

\section{Entrance slit}

The double-slit is illuminated by light entering the apparatus through a narrow entrance slit ${ }^{10}$ in the end-cap of the
PVC pipe. A 5- $\mu \mathrm{m}$ slit width was chosen to reduce the light intensity down into the single photon regime. The slit will act like an effective point source in the horizontal direction provided that it satisfies the coherence condition ${ }^{11}$

$$
L_{\text {source }} \gg \frac{d a}{\lambda}
$$

where $L_{\text {source }}$ is the distance from the source entrance slit to the double-slit, $a$ is the slit width $(=5 \mu \mathrm{m})$, and $d$ is the double-slit separation $(=1 \mathrm{~mm})$. For $\lambda=500 \mathrm{~nm}, L_{\text {source }} \gg$ $1 \mathrm{~cm}$. Our entrance slit distance of $L_{\text {source }}=2 \mathrm{~m}$ readily satisfies the coherence condition and guarantees that the waves emerging from the double-slits are spatially coherent. A discussion of the quantum theory of optical coherence and field correlation functions can be found in Glauber. ${ }^{12}$ The 3-mm length of the entrance slit does not satisfy the condition in the vertical direction, a desirable feature that guarantees the interference pattern will not be complicated by diffraction in the vertical direction, as was the case in our previous apparatus that utilized a $25 \mu \mathrm{m}$ pinhole as the entrance aperture.

\section{Light source}

We have successfully used a low-pressure Na lamp, various LEDs, a diode laser pointer, and an incandescent light bulb. Any light source will do, but our preferred source is a blue LED. ${ }^{13}$ It is physically small, uses three AA batteries for power, and its color spectrum is narrow enough to act as a monochromatic light source. Mounted $10 \mathrm{~cm}$ from the entrance slit, its diffusing lens send out a $60^{\circ}$ cone of light, making alignment a non-issue. A linear polarizing filter ${ }^{14}$ between the LED and the slit reduces the light intensity by a factor of 0.42 . Figure 2 shows the light source and entrance slit. It is efficacy as a single-photon source is addressed in Sec. IV A.

\section{E. Which-path marker}

The which-path marker consists of two, mutually perpendicular, polarizing filters. ${ }^{15}$ With one oriented vertically and the other horizontally, they are butted together side-by-side and held in place using a standard lens holder. ${ }^{16}$ Positioned closely in front of the double slit, this dual filter mask can be adjusted so that there is (1) no filter in front of the slits, (2) a vertical filter over both slits, (3) a vertical filter over one slit and a horizontal filter over the other, or (4) a horizontal filter over both slits. Since the separation of the double-slit is only $1 \mathrm{~mm}$, one needs to be able to precisely and reproducibly position the mask. An optics post holder with vernier adjustment was used for this purpose; it can travel $25 \mathrm{~mm}$ with a $0.1-\mathrm{mm}$ resolution. The entire assembly is shown in Fig. 3.

When either the vertical or the horizontal filter covers both slits, the double-slit interference pattern is preserved, albeit at a reduced intensity compared to no filter. When the vertical filter covers one slit and the horizontal filter covers the other, the double-slit pattern disappears completely. Two superimposed single-slit patterns are all that remain. This new arrangement changes the setup into a which-path experiment in the sense that it is now (in principle) possible to know which slit the photon passed through; this destroys the quantum interference.

Introducing a third polarizing filter, the quantum eraser, between the marker and the detector thwarts the which-path experiment if it is oriented $45^{\circ}$ with respect to the marker 
filters. Every photon reaching the detector is now polarized in the direction of the third polarizer and it is no longer possible to know which slit each photon passes through; as a result, the interference phenomenon is restored. The marker and eraser assemblies are shown in Fig. 4.

As in the case of the classical wave version described in Sec. II B, it should be noted that the polarizing filter between the light source and entrance slit is essential for the quantum eraser aspect of the experiment to work. Photons entering the apparatus are polarized at $45^{\circ}$ with respect to each of the which-path polarizing filters. Thus, the probability of a photon emerging from either slit is $50 \%$. Whether or not a photon gets absorbed or makes it through either slit is random, but the probability is 50/50 for each slit. One could not secure complete double-slit interference if there was an imbalance in the number of photons emerging from the slits. ${ }^{17}$ The polarizing filter between the light source and entrance slit is not necessary for the simpler experiment that does not involve the whichpath aspect. In this case, the probability of any photon making it through either slit is the same, regardless of its polarization.

\section{RESULTS AND DISCUSSION}

\section{A. Single photon or not?}

In our previous paper ${ }^{1}$ we pointed out that, strictly speaking, we are not detecting single photons of light but rather single photoelectrons liberated by the light impinging on the detector; this is still true in the present experiment. Furthermore, the detection of a photoelectron does not necessarily imply that a single photon arrived. Light from thermal sources, such as light bulbs, or coherent sources, such as lasers, is characterized by a distribution of photon numbers. When using an extremely attenuated beam of light, there may be on average a single photon in the apparatus, but the statistical properties of light result in a small probability that two or more photons arrive together. ${ }^{18}$ Such a coincidence has come to be known as "bunching" and is characteristic of all classical light fields.

For a true single-photon source, the probability of two or more photons arriving together is zero; this is referred to as "anti-bunching." To verify anti-bunching, one could send the detector signal to a multichannel scaler and create a histogram of the number of photons detected as a function of the time interval between different photons. The number should go to one as the time interval goes to zero. Unfortunately, such a direct measurement is impossible due to detector and electronic instrumentation dead-time limitations. Instead, a 50/50 beam splitter and two detectors can be employed to measure the intensities of the split beams. ${ }^{19}$ For low count rates, these intensities can be used to determine the probability of detecting a photon at time $\tau$ in one detector, conditional on detection of a photon at time 0 in the other, as given by the normalized second-order correlation function $g^{(2)}$ :

$$
g^{(2)}(\tau)=\frac{G^{(2)}(\tau)}{\left|G^{(1)}(0)\right|^{2}}=\frac{\langle I(\tau) I(0)\rangle}{|\langle I\rangle|^{2}},
$$

where the two-time expectation value for the intensity is normalized to the overall intensity $\langle I\rangle .^{20}$

In the case of a single-photon source, the photon is either reflected by the beam splitter or not; consequently, $I(\tau)$ goes to zero at $\tau=0$ and $g^{(2)}(0)=0$. On the other hand, classical sources must have $g^{(2)}(0) \geq 1 .^{21}$ Thus, the second-order correlation function provides a way to differentiate between classical and quantum-mechanical light sources. This is one of the most fundamental phenomena in quantum optics (Glauber, Ref. 12).

Development of single-photon sources is currently a vibrant field of research and the nitrogen-vacancy (NV) center in diamond has received considerable attention as a robust, room-temperature source of single photons. A new experiment in our advanced instructional lab can excite and detect a single NV center. This apparatus was used to provide a source of single photons for the Young's double-slit experiment with a beam intensity of 20,000 photons/s. Since the area of the double-slit intercepts about 1/10th of the cross-sectional area of the incident beam, roughly 2000 photons/s are estimated to pass through the slits. This flux was not sufficient to be detected with the Luca ${ }^{\mathrm{EM}}$ camera; even with maximum binning the signal remained buried in the noise. We estimate that a photon flux an order of magnitude higher is necessary for this camera. It is possible that a highend camera operating at much lower temperatures would work, but its price would be prohibitive.

Although the statistics of the Poisson distribution for a classical light source allows for bunching of photons, the probability of measuring two or more bunched photons is very small for an extremely attenuated light source. For example, Pearson and Jackson ${ }^{18}$ claim that their reduced-intensity laser beam is comprised predominantly of single-photon states, even though $g^{(2)}(0)=1$. They report that $99.9 \%$ of their measurements result in single photons as opposed to coincident photons. This result suggests that reduced-intensity laser light is a good single-photon source, a fact that has been utilized in experiments demonstrating interference. ${ }^{22}$ Furthermore, if the wave packet is spread out over an array of detectors, such as our $668 \times 496$ pixel array, the probability that two photons from that single packet are measured in the same pixel is incredibly small. Thus, to a very good approximation, the single-photon contribution to the interference pattern is measured.

\section{B. Results}

Figure 5 shows the events accumulated by the camera after $0.1 \mathrm{~s}$. The graph below the image shows the counts binned
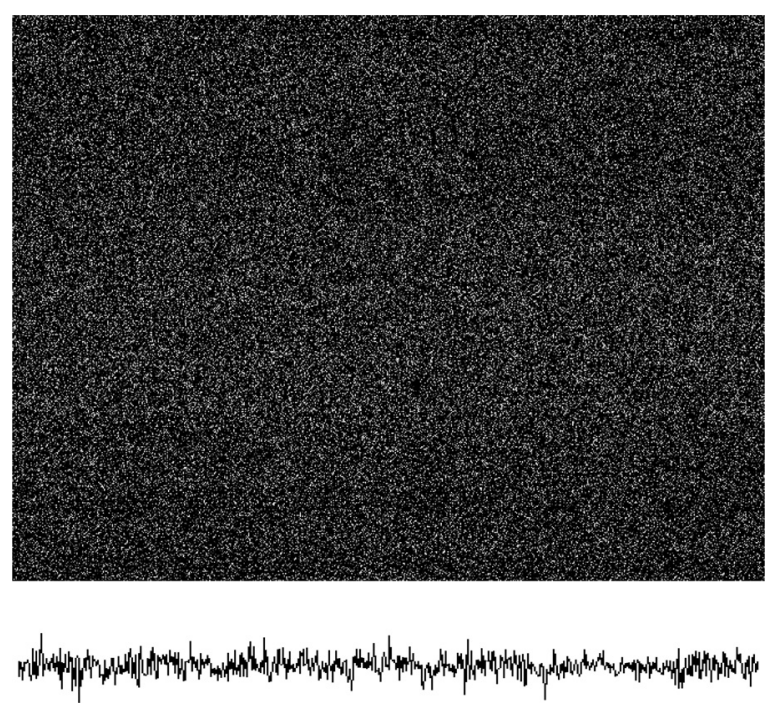

Fig. 5. A 0.1-s exposure; the image grayscale represents a range of 0-20 counts. The graph on the bottom shows the number of counts binned in the vertical direction as a function of horizontal position. Full scale is 20 counts. 

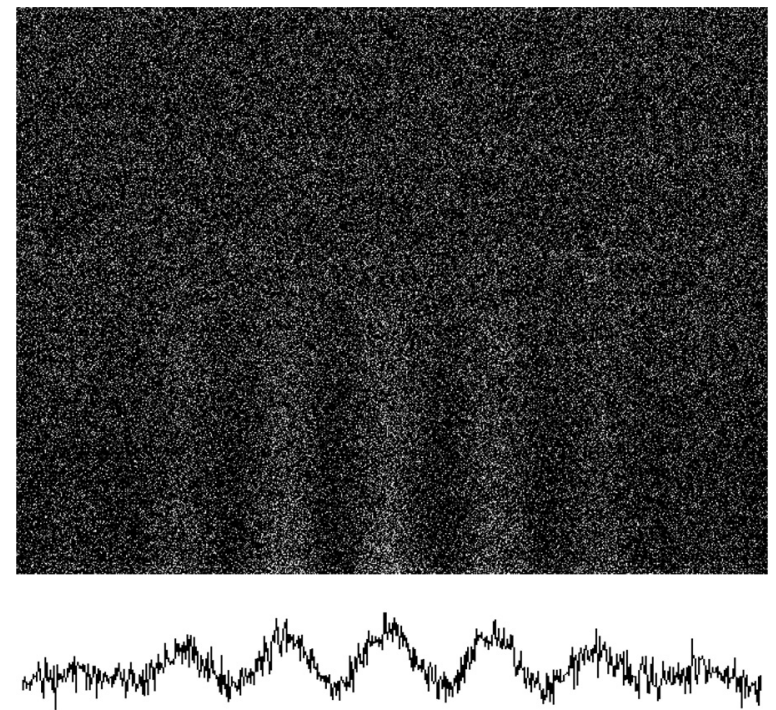

Fig. 6. A 1-s exposure; the graph and image grayscale is $0-20$ counts.

in the vertical direction as a function of the horizontal position. It is clear from the image, as well as the graph, that the arrival of photons at various locations seems to be quite random. Any suggestion otherwise is certainly buried in the "noise." A pattern starts to emerge if enough measurements are amassed; this is evident in Fig. 6, which represents a $1 \mathrm{~s}$ exposure. Given enough measurements the statistics enables us to pull the signal out of the noise. Figure 7 shows the result after $120 \mathrm{~s}$ of accumulation (produced by 240 halfsecond exposures) after approximately 10 million photons have been detected. The probability pattern is clearly evident and looks like the familiar double-slit interference pattern. One can also see that the overall intensity of the double-slit pattern is modulated by the single-slit interference pattern; the central maximum of the single-slit pattern spans the image area of the CCD. The CCD is purposely not centered in the vertical direction on the pattern so that one can compare the image in the lower half with the "background" in
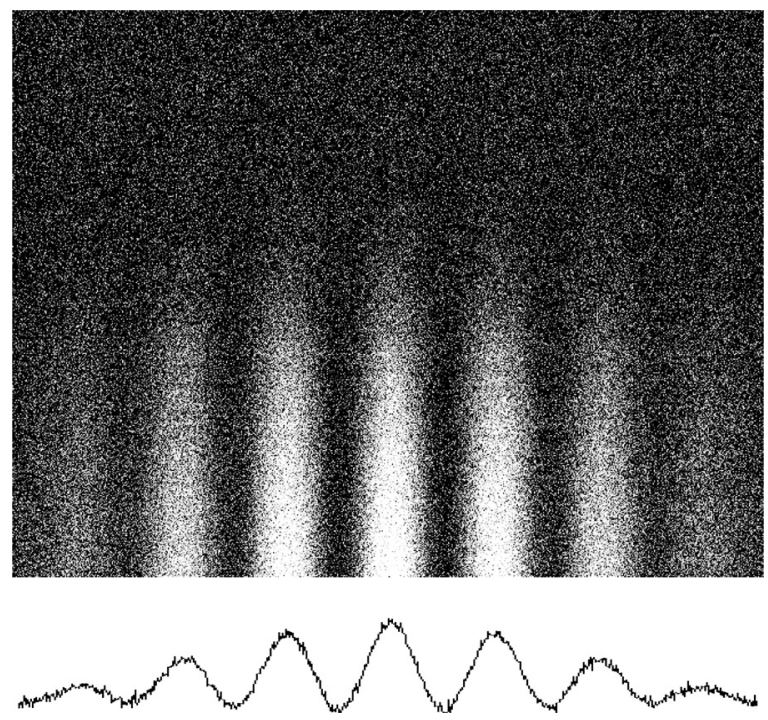

Fig. 7. The sum of 240 half-second exposures (120s accumulation time). The graph and image grayscale is $0-500$ counts.

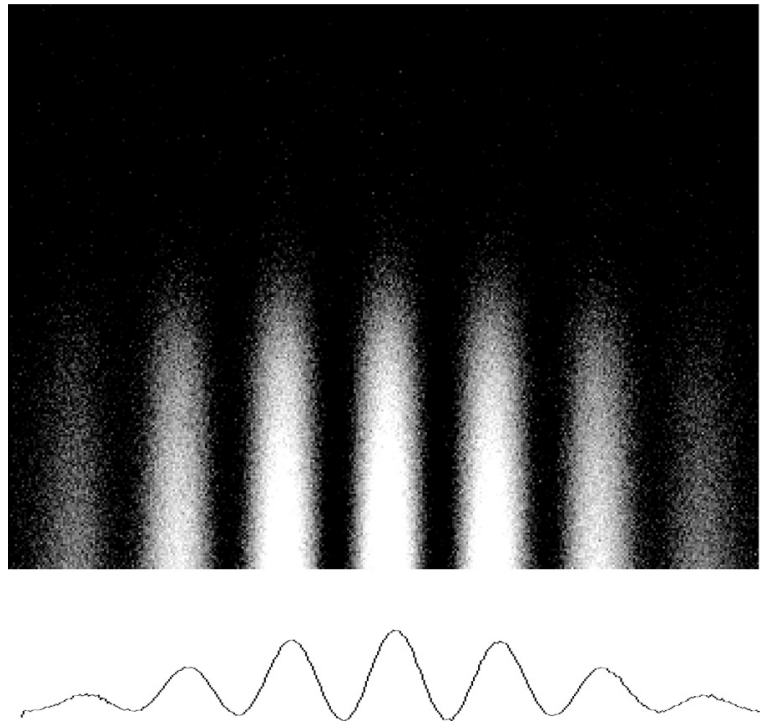

Fig. 8. The sum of 240 half-second exposures ( $120 \mathrm{~s}$ accumulation time) with $2 \times 2$ binning. The graph and image grayscale is $0-2000$ counts.

the upper half. We include Fig. 8 to show the effect of $2 \times 2$ binning; it also portrays $120 \mathrm{~s}$ total accumulation time, but the signal to noise ratio is dramatically improved (see Appendix A).

Figure 9 shows the result of placing the Polaroid marker in front of the double slit. The double-slit interference pattern is completely gone and replaced by the central maximum of the single-slit pattern. Again, offsetting the image on the CCD makes it visually easier to identify the single-slit pattern. The $2 \times 2$ binning technique was used to enhance the signal to noise ratio. Figure 10 shows that the double-slit pattern is restored when the quantum eraser is inserted before the photons reach the detector.

To demonstrate that any light source can be used for this experiment we show in Fig. 11 an image obtained using an incandescent light bulb. Only three distinct maxima are

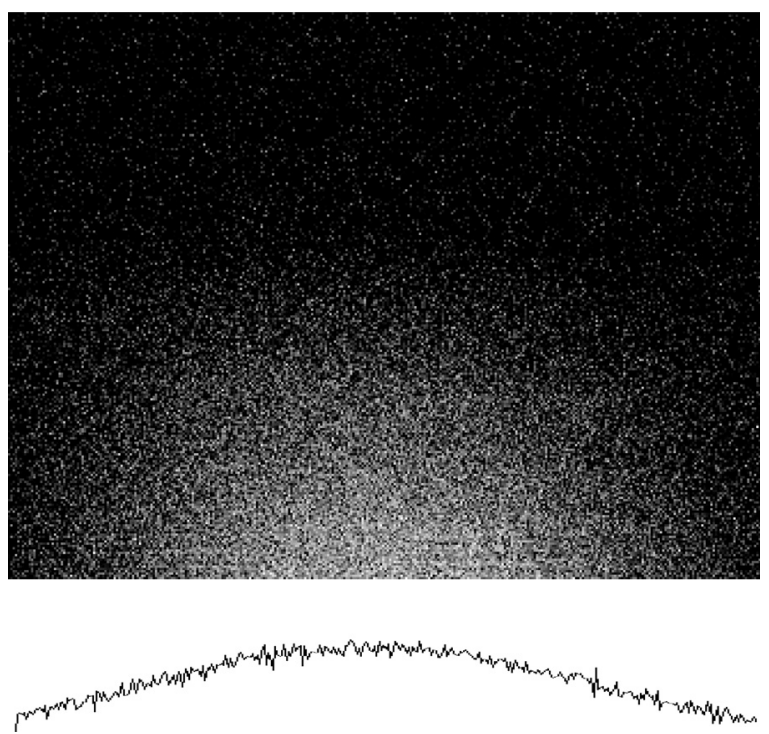

Fig. 9. With the marker in place, the double-slit interference disappears. The graph and image grayscale is $0-1000$ counts. The image shows the sum of 240 half-second exposures for a total of $120 \mathrm{~s}$ of accumulation time with $2 \times 2$ binning. 

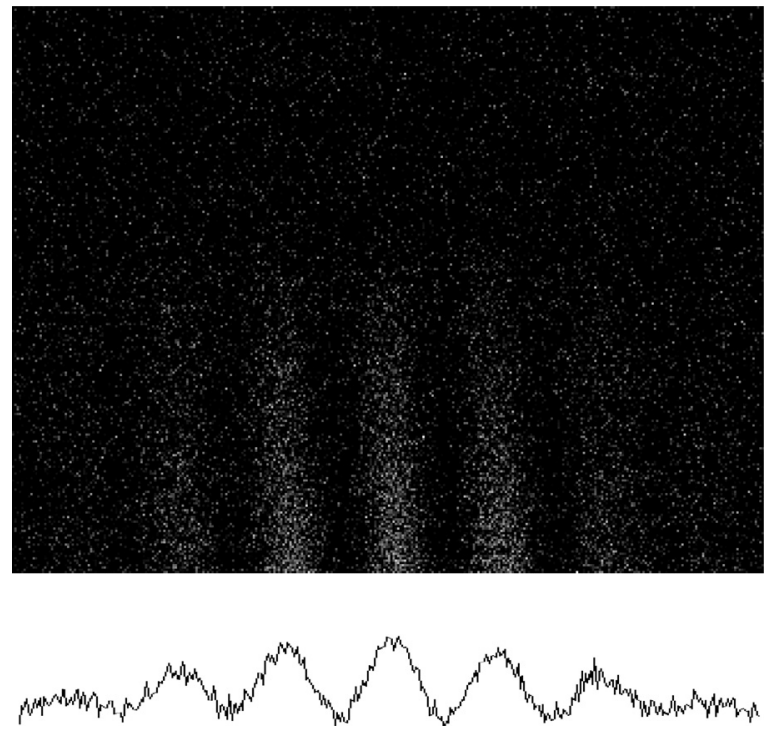

Fig. 10. With the quantum eraser in place, the double-slit interference is restored. The graph and image grayscale is $0-1000$ counts.

evident compared with seven maxima for the blue LED. This is a result of the superposition of the interference patterns from a continuum of wavelengths across the visible spectrum. All these patterns have the same central maximum, but the pattern is more spread out for the longer the wavelengths; this smears out the higher-order maxima and minima. ${ }^{23}$ Even the blue LED interference pattern shows signs of a little smearing at the higher order maxima since the light is not purely monochromatic. A Sodium light source $(\lambda=589.0$ and $589.6 \mathrm{~nm}$ ) produces a very clean pattern with five maxima spanning the CCD as shown in Fig. 12.

One can use Fig. 12 data for a quantitative student exercise. For example, knowing the wavelength and determining from the graph that the maxima are $115 \pm 1$ pixels apart, one can use the measured spacing of the blue LED maxima (92 pixels) to calculate the wavelength of the blue LED. Another possibility is to use the fact that the 658-pixel CCD array is
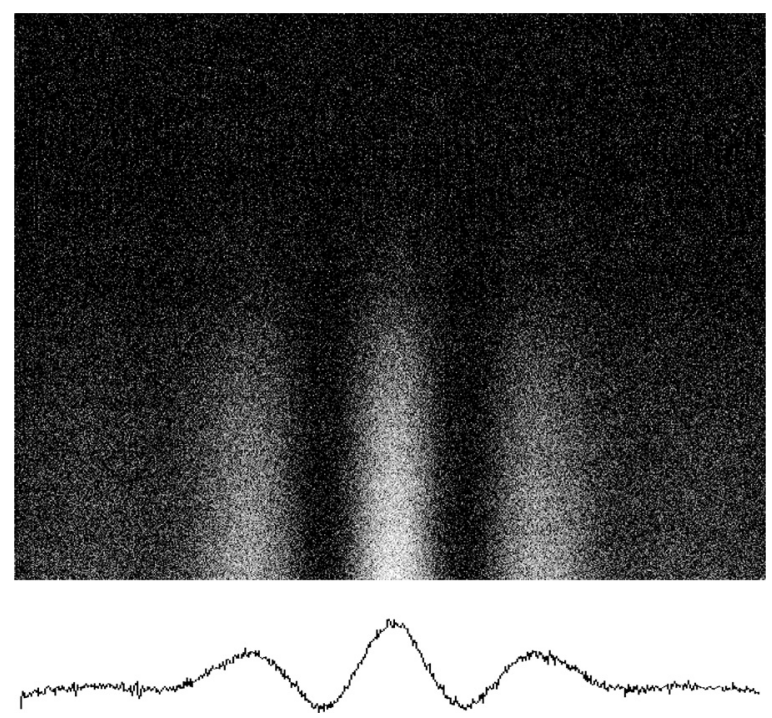

Fig. 11. Double-slit interference with an incandescent light source. The graph and image grayscale is $0-1000$ counts.

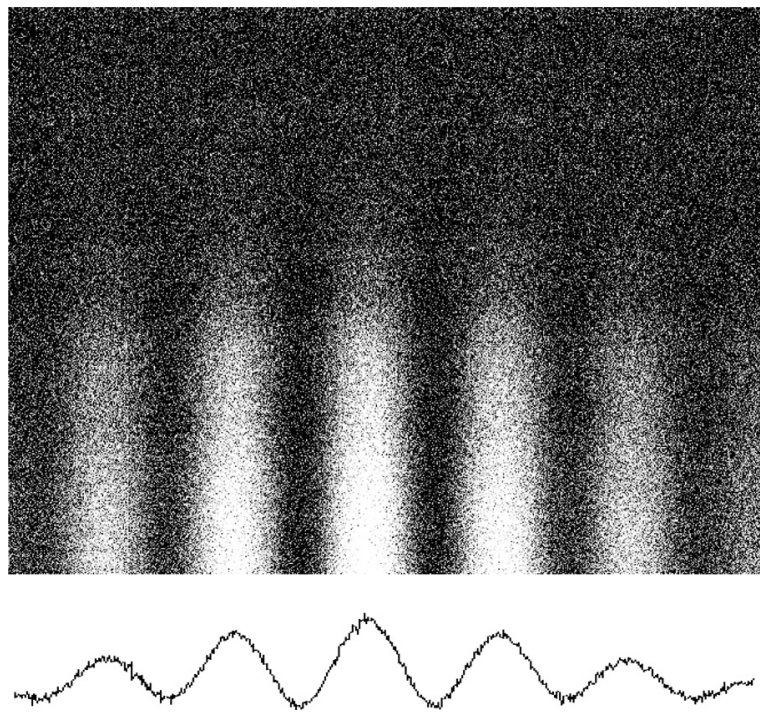

Fig. 12. Double-slit interference with a low-pressure Na light source. The graph and image grayscale is $0-500$ counts.

$6.58 \mathrm{~mm}$ wide to calculate other parameters such as slit separation, width, or CCD distance.

Compared to classical physics, modern physics is not as well represented in the repertoire of demonstration experiments and this experiment helps rectify the imbalance. The single photon double-slit experiment seems to have become a paradigm for quantum mechanics as it is taught in introductory courses, making this experiment an important lecture-demonstration.

\section{ACKNOWLEDGMENTS}

The authors would like to acknowledge our anonymous referees for their helpful comments, especially their suggestions to address the issue of whether or not this is truly a single-photon experiment.

\section{APPENDIX A: NOISE SOURCES IN A CCD IMAGE}

When choosing the right camera for this experiment, quantum efficiency is not the only parameter to take into consideration. CCD cameras work by absorbing photons to generate electrons in a metal oxide silicon capacitor. Each pixel in a CCD is a tiny MOS capacitor. The number of electrons stored in each pixel "well" is proportional to the number of photons that struck the pixel. Precisely counting the number of electrons would tell you how many photons struck the pixel. In the real world, the process of converting light to pixel values in a CCD image is governed by fundamental physics and other factors that introduce "noise" into the final image. The noise manifests itself as variations in pixel values that make the image a less-than-exact representation of the original variation in light.

\section{Noise in the signal itself}

The detection of photons by the CCD is a statistical process and the low count-rate follows a Poisson distribution. The deviation in the light intensity detected by each pixel is proportional to the square root of the number of photons detected by that pixel. 


\section{Thermally generated noise}

Dark-current is generated by thermally excited electrons in the silicon substrate of the CCD. This so-called thermal noise (or dark noise) increases with the length of the exposure, and the scatter is equal to the square root of the number of thermal electrons knocked free in each pixel. The simplest way to reduce the dark noise is to cool the CCD. With thermoelectric cooling - the Luca operates at $-20{ }^{\circ} \mathrm{C}$ - the dark noise can be reduced to negligible levels (typically $0.05 e^{-}$ per pixel/s).

\section{Pixel non-uniformity}

Individual pixels are not perfect and have slightly different sensitivities to light. Some pixels even appear "hot," with as many as three times the number of counts above background. As long as these imperfections stay constant in time, they can be recorded and subtracted from the image.

\section{Readout noise}

The ultimate noise limit of the CCD is determined by the readout noise. Readout noise is generated by the electronic circuitry that converts the charge from each pixel into a digitized light intensity in the final image. One contribution to this noise comes from shifting the accumulated charges on the CCD chip register-electrons can be left behind or jump ahead. Another contribution comes from the on-chip amplifier that converts the number of electrons into an analog voltage. All amplifiers generate their own noise, although welldesigned electronic circuits can minimize the amount of random noise introduced. Finally, the amplifier output voltage is converted into a number that is passed on to the computer software as the pixel's value. This A/D conversion also contributes noise. EMCCDs use a conventional CCD shift register extended with an additional gain register before readout. The gain register is operated at higher voltages, resulting in "impact ionizations" when electrons are transferred. The secondary electrons produced by impact ionization results in multiplication of the signal above the read noise of the detector. Using an Optronic Laboratories model 310 Low Light Level Calibration Std., we determined that an electron multiplication gain of 35 was optimum for the present experiment.

\section{Software noise reduction}

Because most of the noise is from readout, and the EMCCD is read out hundreds of times during the experiment, we use a software "trick" to subtract the background noise from each exposure. First, a background file is created by taking exposures with the light source off and reading out these exposures as many times as intended during the experiment. These exposures are then added together and the resulting data file is divided by the number of exposures. This procedure gives us an average background file that is subtracted from each exposure taken during the experiment. The "hot" pixels, mentioned above, are completely eliminated with this technique.

Binning is another tool at our disposal to increase the signal to noise ratio. Binning is a process that combines charges from two or more pixels on the EMCCD chip prior to readout. Summing charges on the EMCCD and doing a single readout gives better noise performance than reading out the individual pixels and then summing them in the computer memory. The quantum eraser experiment greatly benefits from binning. The combination of the which-path marker plus the quantum eraser polarizer reduces the intensity to approximately $20 \%$ of its already low value, and this really pushes the capabilities of the camera. Although the restored double-slit pattern is visible without binning, utilizing $2 \times 2$ binning brings out the pattern beautifully.

\section{APPENDIX B: ACQUISITION WITH THE EMCCD}

Camera functionalities as well as display options are software controlled; these include exposure length, number of exposures, binning, and electron multiplication. Several software platforms are available. ${ }^{24}$ For our measurements, we used Andor's Solis. Solis' graphical user interface is easy to use for camera setup and single image acquisition. We run an Andor Basic script, shown below, within Solis to accumulate images and subtract an averaged background image. To streamline presentation, typical parameters such as exposure time and number of images are embedded in the program. We gratefully acknowledge programming help from the Andor technical staff.

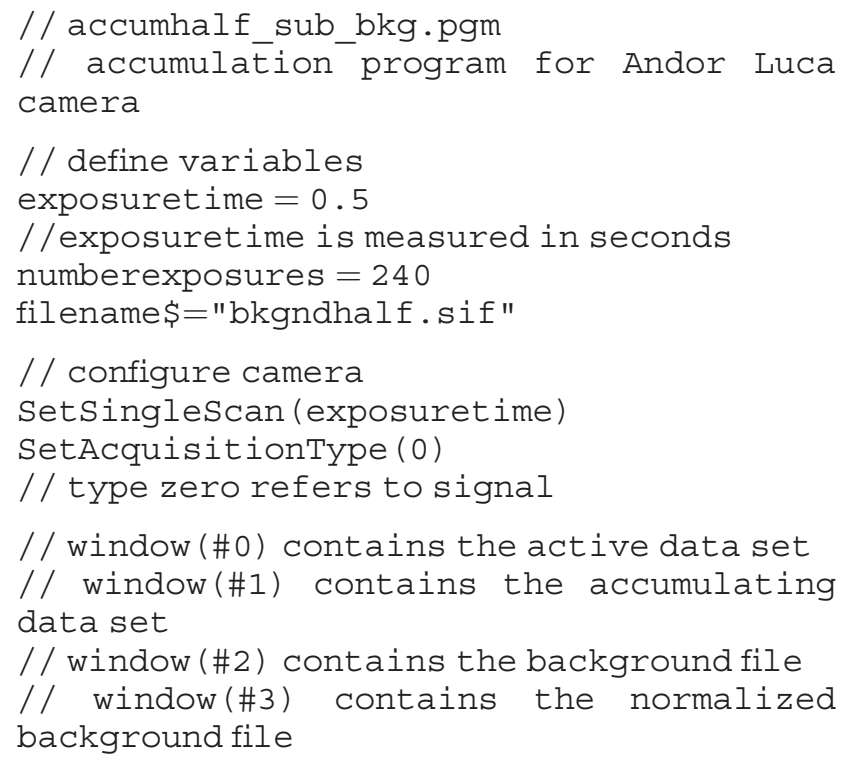


//accumulate and subtract background image

$\# 1=\# 1+(\# 0$ sig - \#3)

//maximize the accumulation window MaximizeWindow (\#1)

Cls ( )

print ("Acquisition ";i;" of ";

numberexposures)

$i=i+1$

wend

CloseWindow (\#2)

CloseWindow (\#3)

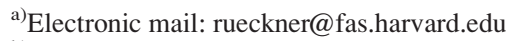

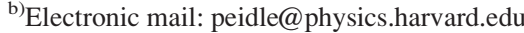

${ }^{1} \mathrm{~W}$. Rueckner and P. Titcomb, "A lecture demonstration of single photon interference,” Am. J. Phys. 64(2), 184-188 (1996).

${ }^{2}$ S. P. Walborn, M. O. Terra Cunha, S. Pádua, and C. H. Monken, "Doubleslit quantum eraser," Phys. Rev. A 65(3), 033818-1-6 (2002).

${ }^{3}$ A. Hobson, "Teaching Quantum Theory in the Introductory Course," Phys. Teacher 34, 202-210 (1996).

${ }^{4}$ Interference is not $100 \%$ complete at all other incident polarization orientations and non-existent near vertical and horizontal orientations. Thus, for unpolarized light, what interference there is near $45^{\circ}$ gets swamped and washed out by the stronger single-slit pattern from all the other polarization orientations. For example, suppose the incident light is polarized $30^{\circ}$ with respect to the vertical. Malus's law tells us that $1 / 4$ of the incident intensity emanates from the slit with horizontal polarizer and 3/4 of the incident intensity from the other slit with vertical polarizer. One cannot hope to secure complete destructive interference with a factor of three difference in intensities.

${ }^{5}$ R. Hillmer and P. Kwiat, "A Do-It-Yourself Quantum Eraser," Sci. Am. 296, 90-95 (2007).

${ }^{6}$ Andor Technology Luca ${ }^{\mathrm{EM}}$ DL-658M monochrome (website <http:// andor.com $>$ ). Although not as prohibitively expensive as the camera previously used, with a price tag of $\$ 10,000$ this is a high-priced item, especially if it were procured for a single purpose. Such a serious investment can be rationalized by making use of the camera in other ways. For example, we share the camera with our instructional physics labs for use in other optics experiments. Furthermore, demo models are sometimes available at a discount-contact your local representative for details on what might be available. The manufacturer's 50\% QE claim was checked and corroborated by illuminating the CCD with an Optronic Laboratories model 310 Low Light Level Calibration Std., (on loan from the Harvard Smithsonian).

${ }^{7}$ Andor Solis Windows 2000 based package with functionality for data acquisition and processing.

${ }^{8}$ National Aperture, Inc. Salem, NH, <http://nationalaperture.com/slits.htm >.
${ }^{9}$ Frank S. Crawford, Jr., Waves, Berkeley Physics Course-vol. 3, (McGraw-Hill, NY, 1968), pp. 456-459.

${ }^{10}$ National Aperture, Inc. Salem, $\mathrm{NH}<$ http://nationalaperture.com/slits.htm $>$.

${ }^{11}$ Frank S. Crawford, Jr., Waves, Berkeley Physics Course-vol. 3, (McGraw-Hill, NY, 1968), pp. 470-473.

${ }^{12}$ R. J. Glauber, "Nobel Lecture: One hundred years of light quanta," Rev. Mod. Phys. 78, 1267-1278 (2006).

${ }^{13}$ Lumex SSL-LX5093USBD: $470 \mathrm{~nm}, 300 \mathrm{mcd}$.

${ }^{14} \mathrm{HN} 42 \mathrm{HE} \times .011^{\prime \prime}(0.28-\mathrm{mm}$ thick) sheet polarizer (HN50 would be an "ideal" polarizer with $50 \%$ transmission). Formerly made by Polaroid, this material is now made by $3 \mathrm{M}$ under the name Vikuiti.

${ }^{15} \mathrm{HN} 42 \mathrm{HE}$ was chosen for its combination of high transmittance and high extinction: $42 \%$ transmission for a single sheet and $0.002 \%$ transmission for two (crossed) sheets at $90^{\circ}$. A newer type (HN38S) is even better in this respect: $38 \%$ and $0.0004 \%$. (One might guess that the S stands for "super.")

${ }^{16}$ Newport LH-1 fixed lens mount $<$ http://newport.com $>$.

${ }^{17} \mathrm{We}$ found this to be the case using a low-pressure Na lamp and LED. These light sources are normally considered to be unpolarized but they are in fact very slightly polarized due to their construction geometry. Consequently, one can observe a very tiny hint of double-slit interference being restored by the quantum eraser, but it's really overwhelmed by the single-slit pattern.

${ }^{18}$ J. J. Thorn, M. S. Neel, V. W. Donato, G. S. Bergreen, R. E. Davies, and M. Beck, "Observing the quantum behavior of light in an undergraduate laboratory,” Am. J. Phys. 72(9), 1210-1219 (2004); B. J. Pearson and D. P. Jackson, "A hands-on introduction to single photons and quantum mechanics for undergraduates," Am. J. Phys. 78(5), 471-484 (2010). These two papers provide an excellent introduction to the subject matter for the non-specialist with a large number of references to original papers and literature.

${ }^{19} \mathrm{P}$. Grangier, G. Roger, and A. Aspect, "Experimental Evidence for a Photon Anticorrelation Effect on a Beam Splitter: A New Light on SinglePhoton Interferences," Europhys. Lett. 1(4), 173-179 (1986).

${ }^{20}$ L. I. Childress, "Coherent manipulation of single quantum systems in the solid state," Ph.D. dissertation, Harvard University (2007), pp. $17-18$.

${ }^{21}$ D. F. Walls and G. J. Milburn, Quantum Optics, 2nd ed. (Springer-Verlag, Berlin, 2008), pp. 38-42; L. Mandel and E. Wolf, Optical Coherence and Quantum Optics (Cambridge U.P., Berlin, 1995), pp. 622-627, 639-643, and 712-723.

${ }^{22}$ M. B. Schneider and I. A. LaPuma, "A simple experiment for discussion of quantum interference and which-way measurement," Am. J. Phys. 70(3), 266-271 (2002); T. L. Dimitrova and A. Weis, "The wave-particle duality of light: A demonstration experiment," Am. J. Phys. 76(2), 137-142 (2008). Both of these experiments use a laser for the light source.

${ }^{23}$ For example, the second position where one obtains destructive interference for red light very closely coincides with the second maximum (constructive interference) for blue light.

${ }^{24}$ Four software packages are described at $<$ http://andor.com/products/ software $>$.

\section{MAKE YOUR ONLINE MANUSCRIPTS COME ALIVE}

If a picture is worth a thousand words, videos or animation may be worth a million. If you submit a manuscript that includes an experiment or computer simulation, why not make a video clip of the experiment or an animation of the simulation. These files can be placed on the Supplementary Material server with a direct link from your manuscript. In addition, video files can be directly linked to the online version of your article, giving readers instant access to your movies and adding significant value to your article.

See http://ajp.dickinson.edu/Contributors/EPAPS.html for more information. 